\title{
Tratamiento ambulatorio de pacientes asmáticos en crisis: comparación de dos esquemas terapéuticos
}

\author{
Dr. Mario Calvo G. ${ }^{1}$ y Dra. L. Escobaro. ${ }^{2}$
}

\author{
AMBULATORY TREATMENT OF PATIENTS WITH ASTHMATIC CRISIS COMPARISSON \\ OF TWO THERAPEUTIC REGIMENS
}

\begin{abstract}
A single blind prospective study of tittw imbulator patients with crisis of hronchial isthntit is presented; twenty five of then were treated whth subcutaneuos attrenaline, followerl by oral eferdrine and twenty five whth Fenoterol sinup.

The clinical picture and laburatory data response of beth groujs were andized, resulting in significant statistical differences.

According with national and intemational experiences, feuoterol was found to be a goud altemitive drug in the treatment of acute asthmatic crisis, particularly the to its rapid browchodilator effect, and the abscense of important actuerse reactions.
\end{abstract}

El asma bronquial, una de las afecciones crónicas más frecuentes en la infancia, constituye para el Pediaba un motivo diario de consulta que no siempre se resuelve lavorablemente.

Su prevalencia varia segín diversos autores desde un 3,5 por mil ${ }^{1}$ hasta 3 y $10 \%$, dependiendo esta diferencia de los criterios de definición usados y probablemente de factores geográficos.

En la literatura nacional y extranjera existen múltiples trabajos relacionados con el uso de broncodilatadores, nanejo intrahospitalario y prevención del asma bron segu imiento y manejo ambulatorio de las crisis.

En nuestro hospital, las consultas por asmit broncpuial tanto en policlinica como en servicios de urgencia, constituyen una fuerte carga asistencial, it igial que en los servicios de hospitalización.

Estos hechos nos motivaron a efectuar un seguimiento ambulatorio de los pacientes con crisis asmática y al mismo tiempo comparar la efectividad de dos esquemas terapéuticos diferentes.

\section{MATERLAL Y METODOS}

Se programi un estudio prospectivo con una muestra de 50 pacientes portadores de asma bronquial en crisis, que consultaron en la policlínica de Enfermedades Respiratorias del Servicio de

\footnotetext{
I. M édien Pediatra. Institulo de J'exliatrín, Facultanl ste Medicina, Liniversidad Austral de Chile.

${ }^{2}$ Medico Becario. Servicio de Pediatria. Hospital Regional de Valdivia.
}

Pediatría del Hospital Regional de Vaddivia; la que se cumplió desde junio a septiembre de 1981.

Para los fînes de este trabajo se definio en crisis a un paciente portador de asma bronquial que consultó con evidencias clínicas de obstrucción bronguial y una caída del FEM (flujo espiratorio máximo) mayor del $20 \%$ del valor teórico normal para su estatura y edad.

Se diseñó una fỉcha estaindar para evaluación de estos pacientes, registríndose: edad, frecuencia cardíaca y respiratoria, retracción de partes blandas, sibilancias y flujo espiratorio máximo como indicadores globales de dificultad respiratoria. Estos datos se registraron previos a la administración del medicamento y a los 5, 15, 30 y 60 minutos; 24, 48, 72 y 96 horas después de iniciada la terapia. Se consignó además, el uso de otros medicamentos tales como antibióticos, expectorantes y/o antitérmicos y los efectos adversos observados.

Para evitar la importancia que pudiera tener el grado de subjetividad en la medición de las variables clínicas, todos los pacientes fueron evaluados por un solo médico, midiéndose además, el flujo espiratorio máximo como elemento de mayor objetividad (medidor de flujo de Wright). ${ }^{9}$

En forma aleatoria se constituyeron dos grupos de 25 pacientes, recibiendo uno de ellos (Grupo A) Fenoterol (Berotec, jarabe M.R.), 0.25 mgs. /Kg./24 hrs, fraccionado cada 8 horas por via oral y el otro (Grupo B), Adrenalina subcutánea 0.01 mgs/ $\mathrm{K} g / \mathrm{l}$ vez, continuando con Efedrina (3 mgs/ $\mathrm{Kg} / 24 \mathrm{hrs}$. cada 8 hrs. vía oral).

Las otras medidas terapéuticas (antitémicos, 
antibióticos y/o expectorantes) se indicciron en los casos necesarios en forma independiente de la terapla broncodilatadora.

Los resultados se analizaron con una dócima no paramétrica, Test de Mann Whitney, "ya que la distribución de los datos no fue simétrica. El nivel de significación fue de $1 \%$.

\section{RESULTADOS}

La edad promedio de los 50 pacientes fue de 7 años 6 meses, oscilando entre 2 y 14 años; siendo en el grupo $A$ de 8 años 1 mes y 6 años 5 meses en el grupo B. El 72\% de los pacientes fue de sexo masculino, porcentaje que se mantuvo en ambos grupos.

Seis pacientes (12\%) recibieron antibióticos, distribuy éndose tres en cada grupo.

El promedio de la frecuencia cardíaca basal en el grupo A fue de 103 latidos por minuto, observándose normalización (84 latidos (por minuto) en el momento del alta (descenso de 19\%). En el grupo B fue de 111 y 100 latidos por minuto, respectivamente, con un descenso de $10 \%$.

Las variables más importantes $y$ objetivas fueron las horas de evolución erı regresión de la polipnea, retracción de partes blandas, sibilancias y nomalización del flujo espiratorio máxiruo.

\section{Polipnea (Grálico 1).}

La evolución de este paránetro fue más rípida en el grupo A que en el B, nomializándose el promedio de la frecuencia respiratoria a las 48 y 96 horas respectivamente.

\section{Gráfico 1}

Horas de regresión de polipnea en pacientes asmáticos tratados con fenoterol (Gnıpo A) y adrerıalina inás efedrina (Grupo B).

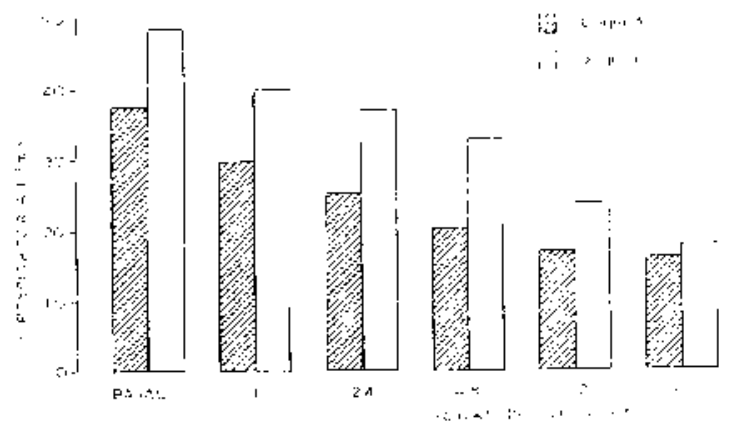

Retracción de portes blandos (Gráfico 2).

Al igual que en el gráfico anterior, se observó menos horas de evolución en el grupo A, normalizándose este signo prácticamente en todos los pacientes a las 24 horas de evolución, lo (jue sólo ocurrió a las 72 horas en el grupo $B$.

\section{Gráfico 2}

Horas de regresión de retracción de partes blandias en pacientes asmáticos tratados con fenoterol (Gripon A) adrenalina más elextrina (Grupı B).

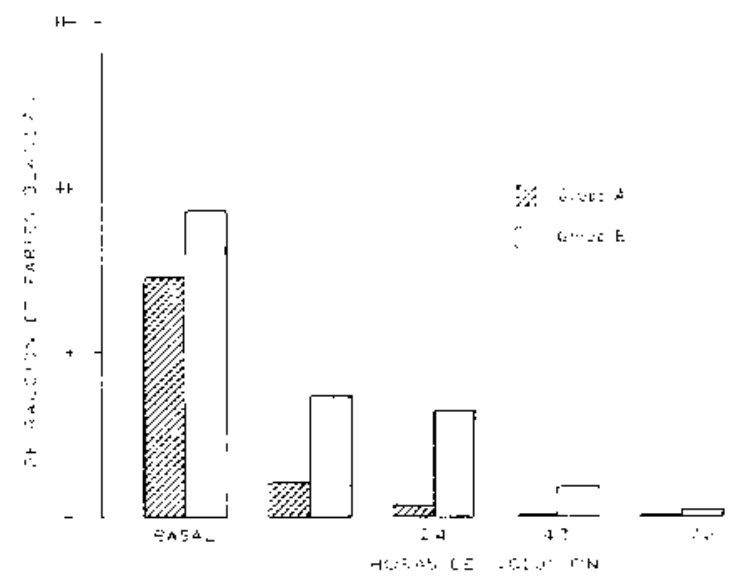

\section{Sibilancias (Gráfico 3).}

Se observó regresión de este signo a las 48 horas de evoltción en el grupo A y a las 72 horas en el B.

\section{Grafico 3}

Horas de regresion de sibilanicias en pacientes asmáti(D) tratades con fenoterol (Grupo A) y adrentina más efedrina (Grupo B).

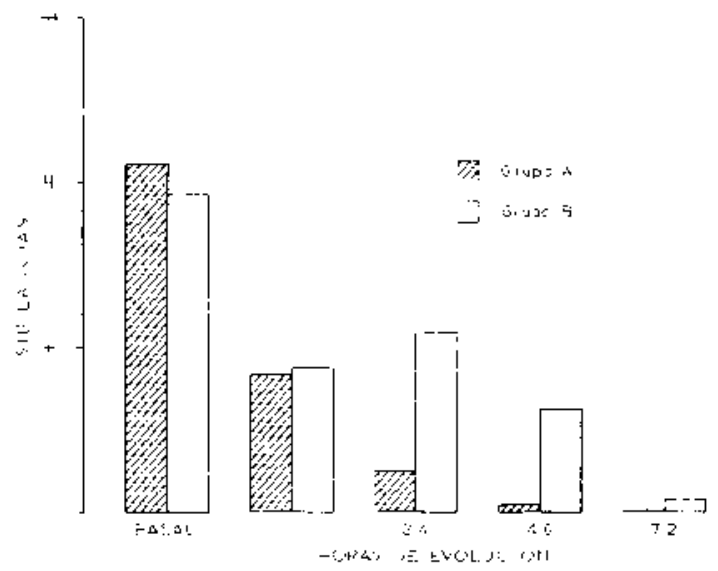

Fiujo espirctorio máximo (FEM) (Grático 4).

Por requerir este método la colaboración del 
paciente, se logró determinar en 35 de ellos, 20 de los cuales eran del grupo A y los 15 restantes del grupo B. Se logró nomalización de este parámetro a las 48 y 96 horas respectivamente.

\section{Gráfico 4}

Horas de normalización de flujo espiratorio máximo en pacientes asmáticos tratados con fenoterol (Gripo A) y adrenalina mäs efedrina (Grupo B).

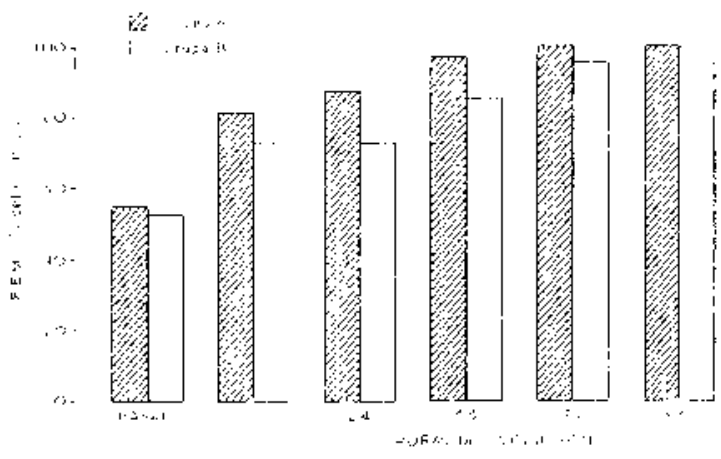

Significacion estodistica de las diferencias (Tabla 1).

En las cuatro variables analizadas, las diferencias encontradas fueron estadisticamente significativas con un nivel de confianza de $99 \%$ $(\mathrm{p}<0.01)$.

\section{Tabla 1}

Significación estadística de las diferencias encontradas en pacientes asmáticos tratados con fenoterol y adrenalina más efédrina.

\begin{tabular}{|c|c|}
\hline Variables & Significación estiadisica \\
\hline Folipnea & $p<0,0 i$ \\
\hline Pietracción de sartes blandas & $p<0,01$ \\
\hline jibularkias & $p<c, 01$ \\
\hline$F . \equiv 4$ & $p<0,01$ \\
\hline
\end{tabular}

\section{Efectos colaterales.}

El fármaco empleado en el grupo A fue muy hien tolerado, no existiendo efectos colaterales. En el gnipo B; 4 pacientes (16\%) presentaron efectos adversos consistentes en taquicardia, irritabilidad, náuseas y vómitos que no obligaron a suspender el medicamento.

\section{COMENTARIO}

En la últirna década ba existido un progreso sustancial en la calidad de las drogas para el tra- tamiento del asma, ya sea para la crisis o como terapia de prevención. En nuestro país y principalmente en el organismo estatal de salud, el costo de los medicamentos $\mathrm{B}_{2}$ específicos ha impedido su uso cotidiano; manteniéndose aún drogas de efecto $\alpha$ y $\beta$ adrenérgicos, en uso desde hace más de 50 años. ${ }^{6,7.11}$

Si bien es cierto ambos grupos de medicanentos tienen por acción fundamental aumentar el calibre de la vía aérea, en base a relajación de la musculatura lisa bronquial, existen diferencias importantes en la potencia del efecto, su selectividad y ios efectos colaterales que presentan.

El uso de Adrenalina subcutánea, como terapia de urgencia en una crisis asmática leve o moderada, para continuar con efedrina oral, se ha recomendado clásicamente en nuestro país. ${ }^{12,13,14}$ En nuestra experiencia la asociación de estas drogas fue útil, por cuanto ninguno de los pacientes requirió hospitalización, observándose ya a los 30 minutos respuesta tanto clínica como de laboratorio. Durante la primera hora de terapia existió junto a los signos de regresión de dificultad respiratoria, un aumento de la frecuencia cardiaca.

La taquicardia inicial, consecuencia del cuadro bronquial obstructivo, aumentó por el efecto $\alpha$ adrenérgico de estos medicamentos; efecto gue se objetiva mejor cuando al término del tratamiento $y$ habiendo pasado la crisis, permanecian con una frecuencia cardíaca en promedio alta ( 100 latidos por minuto).

Al analizar el perịodo de evolución de los pacientes se desprende que 12 de ellos (48\%) se hicieron asintomáticos a las 72 horas de tratamiento, recibiendo $3(12 \%)$ una terapia inás prolongada (96 horas). Ein este grupo no hubo pacientes asintomáticos a las 24 horas de iniciado el tratamiento.

En el grupo tratado con Fenoterol, medicanento $\mathrm{B}_{2}$ altamente selectivo en su efecto sobre la musculatura lisa bronquial $3,4,8,26$ los resultados fueron muy buenos. Pese a la administración por vía oral, se objetivó una respuesta clinica y de latboratorio inicial favorable a los 15 minutos de su administración, haciéndose ésta muxbo más evi. dente a los 30 minutos. La taquicardia inicial, que se debia al cuadro broncinial obstructivo, regresó rápidamente en la medida que cedía la crisis (disininución 19\%) y en el momento del alta la frecuencia cardíaca era nomal. A las dosis usadas de Fenoterol no se observó efecto $\alpha$ adrenérgico, la tolerancia fue excelente $y$ ninguno de los pacientes presentó efectos adversos. 
El elemento más destacable de este medicamento fue la rápida regresión dela signología obstructiva, que permitió que 16 pacientes $\langle 64 \%$ ) estuvieran asintomáticos a las 24 horas de tratamiento y sólo uno de ellos (4\%) a las 72 horas.

Otro hecho destacable del seguimiento ambulatorio de los pacientes fue que ninguno de ellos requirió hospitalización, pese a que algunos consultaron con crisis asmáticas severas. Probablemente el mantener al paciente en reposo y control durante una hora después de iniciada la terapia, más el control posterior es, junto con los broncodilatadores, un factor importante en los buenos resultados obtenidos. Además, si bien es cierto ambos grupos finalmente mejoraron, quedaron demostrados con claridad los beneficios que tiene el uso de fenoterol como droga de prinera línea; beneficios indudables para el paciente y que de ser usado en el sistema nacional de salud, significaria un ahorro importante de recursos humanos dedicadus al control de estos pacientes.

\section{RESUMEN}

Se presenta un estudio prospectivo de seguimiento ambulatorio de $\mathbf{5 0}$ pacientes con Asma Bronquial en crisis. De ellos, 25 recibieron Fenoterol y los restantes Adrenalina subcutánea, por una vez, continuando con Eledrina.

El anćlisis de las variables clínicas y de laboratorio reveló una diferencia estadísticamente significativa $(\mathrm{p}<0.01)$ a favor del grupo que recibió fenoterol.

Se comentan las características de las drogas utilizadas, de acuerdo a la revisión de la literatura nacional y extranjera y la experiencia que se obtiene de este trabajo se concluye que como terapia ambulatoria para crisis asmática, el Fenoterol es un excelente medicamento tanto por la rapidez de su efecto broncodilatador, como por la ausencia de efectos adversos.

\section{REFERENCIAS}

1 Gerbaux, d.: Coureur, d,; Toumier, G. "Patolongia Respiratoria en el niñu", 428. Ed. Salvat, l'. Ed. 1979.

2 Gomez, $G$, "Defituición e importincia del Asma Intantil". Acta Médica. FAB, 1(4): 6-13, Oct. 1979.

3 Shores, S.; Wenbeng, E; Dür, M. "Bucal admintistration of lenoterol aerosol in young children with asthina". S.A. Med. Journal: 50: 1362-1364, 1976.

4 Schurate A.L "Management of acute asthina in childhuod. A randomized evaluation of beta-adrenergic agents". Am. J. Dis. Child.; 134 (5): 474-8, May, 1980).

5 Kanig, P. "Bronchodilators drugs and young children (letteri". Arch. Dic. Child. 54 i 8 ): 649-650, Aug. 1979.

6 Van Arsdes, P.; Poul, G. "Drngas en el manejo del Asma", Bol. Uniom Inter, contra TBC. 53 i.9): 167-177, 1978. Heproducido de Aun. Intem. Med. 87; 68-74, 1977.

7 Galleguillos, F. "Asma Broneuial. Aspectos ctĺnicos e inmuneItigicos". 104-109, Ed. drancibia Hnıs. 14. ed., 1976.

\& Experiencias clínicas nacionides con Berotex. Ptbilicación de Brehringer lngelheinl.

Y Acta Médica. FAB, Erlitorial, 2 (3); 5, Marzo, $15 \times 8$.

10 Domenechl, Missons, J. "Bioestadística. Métoxlos estadísticos parch investigadores". 392-397; Ed. Hender, 14. Ed. 1977.

II Saundes, N, Mcfadden, ER “Asthona-au update". Dis. Month. 24 (11): 36-39, Aug. 1978.

12 Meneghello, d. "Atencisin del nin̄o sano y enlenno". 331 Ed. Andrés Hello, 1978.

13 "Normas de prevención trataniento y comtrol de las enfermedades respiratorias de la inlancia y tulerculosis infentil". Ministerio de Salud. 67-71, Editora Nacional Cabriela Mistrd. Chile, 1974.

14 "Nontas de Pediatria: Enfermedodes Respiratorias y tuberculosís". Ministerio de Salud, 62-67; Ed. Universitaria Chile, 1981.

15 Josephson, G.; Kennedy, H., McKerrie, E., Gibson, G. "Cardiac dysrhythmias during the treatment of acute asthma. A Comparison of two treatnent reginen by a double lilind protocol". Chest. 78 (3): 429-435, Sept. 1980.

16 "Butrntec". Antiasmático y antialérécoco. Puthlicuciones de Bothringer Ingthelheim. 